\title{
Cost-Effectiveness of Adding Androgen Deprivation Therapy to Radiation Therapy for Men with Advanced Prostate Cancer from a U.S. Payer's Perspective
}

\author{
Chi Nguyen, PhD; David R. Lairson, PhD; Michael D. Swartz, PhD; and Xianglin L. Du, MD, PhD
}

\begin{abstract}
BACKGROUND: No study has investigated the cost and effectiveness of androgen deprivation therapy (ADT) and other curative treatment therapies among the Medicare population, and no study has taken into consideration the long-term side effects associated with ADT.

OBJECTIVE: To examine if adding ADT was cost-effective when accounting for ADT-related long-term side effects in men with prostate cancer.

METHODS: For this cost-utility analysis, we used the Surveillance, Epidemiology, and End Results (SEER)-Medicare linked database to estimate and compare patient survival, costs from a health payer's perspective, and cost-effectiveness of 3 treatment modalities for advanced prostate cancer patients, including radiation therapy, radiation plus ADT, and active surveillance. We also estimated quality-adjusted life-years (QALYs) by assigning appropriate health state utility values obtained from the literature for each phase of care and for long-term side effects. Propensity score matching was used to control for bias and confounding that were inherent to the observational study design.
\end{abstract}

RESULTS: Adding ADT to radiation therapy increased median patient survival by 0.71 years. The incremental cost-effectiveness ratio (ICER) for radiation plus ADT versus radiation alone was $\$ 63,049$ and $\$ 295,995$ per mean lifeyear gained for radiation compared with active surveillance, respectively. Treatment-associated adverse side effects substantially reduced QALYs gained. Compared with radiation only, the incremental cost of radiation plus ADT was $\$ 127,900$ per mean QALY and was nearly $80 \%$ cost-effective at a willingness-to-pay threshold of $\$ 210,000$ per QALY.

CONCLUSIONS: Despite ADT-associated costs and long-term side effects, compared with radiation alone, radiation plus ADT was cost-effective at $\$ 127,900$ per QALY.

J Manag Care Spec Pharm. 2019;25(2):225-34

Copyright $\odot 2019$, Academy of Managed Care Pharmacy. All rights reserved.

\section{What is already known about this subject}

Long-term use of androgen deprivation therapy (ADT) is costly, including the cost of therapy and the cost of treating adverse events.

Among all types of ADT, surgical orchiectomy has been found to be the most cost-effective, and the combined androgen blockade is the least economically attractive option.

\section{What this study adds}

This study investigated the cost and effectiveness of ADT and other curative treatment therapies among the Medicare population and took into consideration the long-term side effects associated with ADT.

We conducted a cost-utility analysis of common treatment modalities currently available for high-risk prostate cancer patients and examined 3 strategies: active surveillance, radiation therapy, and the combination of radiation therapy and ADT.

Despite ADT-associated costs and long-term side effects, ADT was cost-effective in men with prostate cancer who received radiation therapy at $\$ 127,900$ per quality-adjusted life-years.

1 rostate cancer is the second most common cancer among men worldwide and is the most common type of cancer among men in the United States. Currently, there are 4 standard treatments available for patients with prostate cancer: radical prostatectomy (surgery), radiation therapy, hormone therapy, and active surveillance. Hormone therapy, also known as androgen deprivation therapy (ADT), can be achieved by either orchiectomy, a surgical procedure to remove the testicles, or with luteinizing hormone-releasing hormone (LHRH) agonists and antagonists to prevent hormone secretion. Leuprolide, Goserelin, Degarelix, and Triptorelin are the LHRH drugs approved for prostate cancer treatment in the United States. ADT helps to slow tumor growth by lowering androgen levels or preventing LHRH from binding to its receptors and consequently causing androgen levels to decline. ADT is recommended to be used in conjunction with radiation therapy among patients who have high-risk prostate cancer or in patients with recurrent or metastatic cancer., ${ }^{1,2}$ ADT can also be applied before external beam radiation to shrink the tumor and make the treatment more effective. However, ADT has been recently viewed as less favorable because of increasing awareness of its associated long-term side effects that may negatively affect patient survival and quality of life (QoL), such as hot flashes, sexual dysfunction, bone fractures, myocardial infarction, and possible cardiac deaths. ${ }^{3-7}$ Another concern is the misuse of ADT among low-risk cancer patients, where the harm may outweigh the benefits. 
Long-term use of ADT is costly because of the cost of therapy and the cost of treating adverse events (AEs). A study that matched cases to noncancer controls found that the initial treatment cost of hormone therapy was $\$ 10,410$ during the first year, and total 5-year cost was $\$ 23,199$. Adding hormone therapy to radiation therapy would increase the cost in the first year to $\$ 17,384$ but not much over the 5-year period after diagnosis, to $\$ 23,488 .{ }^{8}$ Hatoum et al. (2013) reviewed the economic evaluations of hormonal therapy for prostate cancer and found 13 studies published between 1997 and 2011, of which 6 were conducted in the United States. ${ }^{9}$ Most studies in the United States examined the cost-effectiveness of adding ADT to radiation therapy or combining androgen blockade with nonsteroidal antiandrogen or LHRH drugs. ${ }^{10-12}$ Among all types of ADT, surgical orchiectomy was found to be the most cost-effective, and the combined androgen blockade was the least economically attractive option. ${ }^{13}$ Curative therapies such as surgery and radiation were usually compared with active surveillance. A recent study assessed the costs and quality-adjusted life expectancy of active surveillance versus initial treatments for men with localized prostate cancer. Active surveillance was more cost-effective than curative therapies, while brachytherapy was the most effective in terms of quality-adjusted life expectancy and least expensive initial treatment. ${ }^{14}$

To the best of our knowledge, no study has investigated the cost and effectiveness of ADT and other curative treatment therapies among the Medicare population, and no study has taken into consideration the long-term side effects associated with ADT. These issues led to the central questions guiding this study: How did prostate cancer therapies differ in terms of cost and effectiveness? Was adding ADT to radiation therapy costeffective when accounting for ADT-related long-term side effects?

The purpose of this study was to conduct a cost-utility analysis from the perspective of the U.S. Medicare system of common treatment modalities currently available for high-risk (defined as stage III or IV) prostate cancer patients. We examined 3 strategies: active surveillance, radiation therapy, and the combination of radiation therapy and ADT. The comparison of radiation and ADT versus radiation gave insight into the survival benefits of adding ADT to radiation and whether the ADTassociated side effects affected overall QALYs. We included the active surveillance group to examine the baseline survival and QALYs when patients did not receive any curative treatment.

\section{Methods}

\section{Data Sources}

Data for the study were extracted from the Surveillance, Epidemiology, and End Results (SEER)-Medicare linked database..$^{15}$ The SEER program collects data on clinical characteristics, demographics, and causes of death for cancer cases throughout the United States. The SEER data consist of 17 population-based cancer registries, including 7 metropolitan or rural areas (San Francisco/Oakland, Detroit, Atlanta, Seattle, Rural Georgia, Los Angeles County, and the San Jose-Monterey areas) and 8 states (Connecticut, Iowa, New Mexico, Utah, Hawaii, Kentucky, Louisiana, New Jersey), plus Greater California and Greater Georgia areas. The SEER and Medicare data provided information on the date of death, and Medicare data provided the follow-up information from the date of diagnosis to the date of death or date of last follow-up (December 31, 2010).

Medicare is a federally funded program and is the primary health insurer for $97 \%$ of the U.S. population aged 65 years or older. All Medicare beneficiaries receive Part A benefits, and 95\% of beneficiaries subscribe to Part B coverage. The former covers inpatient hospitalizations and care in skilled nursing homes, while the latter covers physicians' services, hospital outpatient services, hospice care, and other outpatient medical services such as diagnostic X-rays and laboratory tests.

\section{Study Design and Study Population}

This was a retrospective, longitudinal, matched-cohort study. We identified 354,701 prostate cancer patients from the SEERMedicare linked database who were 66 years or older and diagnosed with prostate cancer between January 1992 and December 2009 for study eligibility. Patients were followed up to 17 years from diagnosis to the date of death or the end of the study (December 31, 2010), whichever came first.

Patients who were aged 65 years at cancer diagnosis were excluded, since their medical claims 1 year before cancer diagnosis were not available in the Medicare data to ascertain the baseline comorbidity index. Patients who had prostate cancer identified by autopsy or in the death certificate, died within 6 months of diagnosis, had concurrent secondary cancer, had race/ethnicity or month of diagnosis missing, or received any form of hormone therapy before cancer diagnosis were omitted from the analysis. We also excluded men who did not have continuous enrollment in both Part A and Part B or who were enrolled in a health maintenance organization 1 year before and 1 year after diagnosis, since their claims might not have been processed through Medicare.

The final cohort included 8,001 patients who were diagnosed with stage III or stage IV prostate cancer and who underwent either radiation therapy, radiation therapy plus ADT, or active surveillance. The treatments were ascertained using Medicare claim codes within 12 months following diagnosis. We used a propensity score to match patients receiving radiation therapy plus ADT to those in the radiation-only and active surveillance groups.

\section{Effectiveness}

The outcomes were life-years and quality-adjusted life-years (QALYs). The overall survival time was obtained from SEER data, defined as the time from diagnostic date to date of death or the last date of follow-up (December 31, 2010). Patients who 


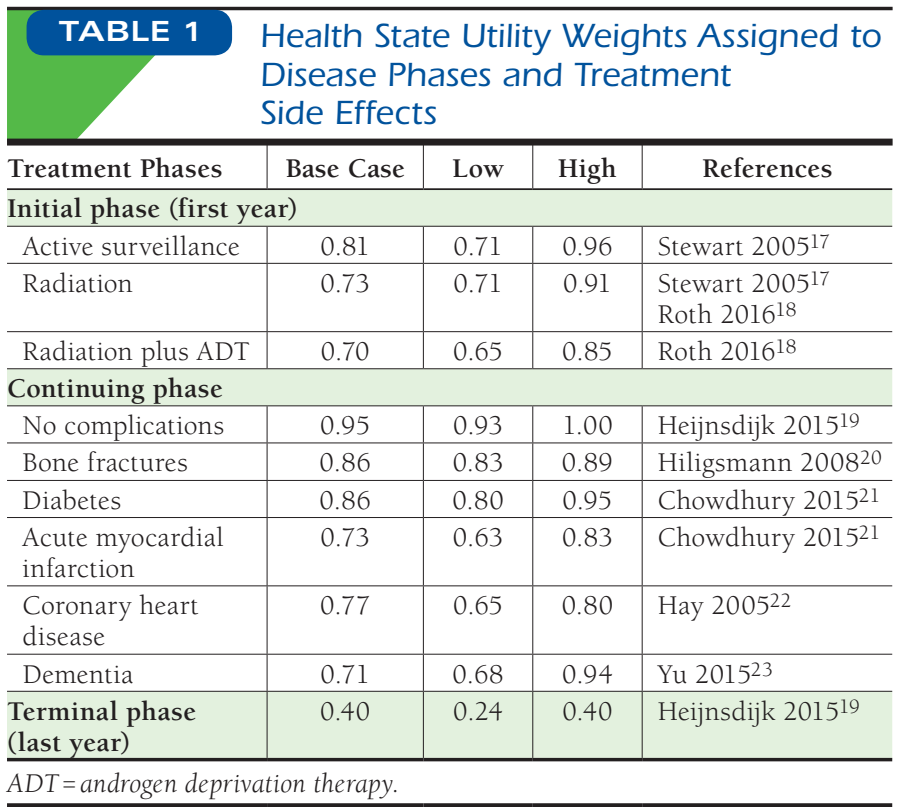

were alive by the last day of follow-up were censored.

The follow-up period was divided into 3 phases: initial, continuing, and terminal. The initial phase was defined as the first year after diagnosis; the terminal phase was the last year of life; and the continuing phase was the duration between the initial phase and terminal phase. For patients who did not survive for 12 months after diagnosis, the entire follow-up period was assigned to the terminal phase. For those with survival between 12 and 24 months, 12 months were assigned to the terminal phase and the rest was initial phase.

The health state utilities for each phase of care and longterm AEs were obtained from a literature review. ${ }^{3-7}$ The health state utilities for the initial phase were weighted by short-term treatment discomfort that a patient experienced after treatment. Continuing-phase utilities were dependent on long-term adverse side effects, and if a patient had multiple adverse conditions, the lowest utility was used. ${ }^{17}$ The common long-term side effects associated with ADT were identified from the literature and included bone fractures, diabetes, acute myocardial infarction, coronary heart disease, and dementia. ${ }^{3-7}$ The methods of assigning health state utilities were widely used among studies using administrative claims data where patient QoL was not available. ${ }^{16}$ Table 1 presents the health utilities assigned to disease phases and treatment side effects. ${ }^{17-23}$

\section{Lifetime Costs}

The direct expenditures for each patient, including inpatient care, short- and long-term hospital stays, and skilled nursing facilities, were ascertained from Part A claims and physician services, and outpatient care was ascertained from Part B claims. Indirect costs such as lost productivity due to treatment visits, transportation, and caregivers were not considered. The costs were estimated according to clinically identifiable phases of care, including initial, continuing, and terminal phases.

Since the costs in Medicare data were the actual dollar payment recorded in the year that payment was made, the costs were adjusted for inflation using the medical care component of the Consumer Price Index and calculated in terms of 2016 U.S. dollars. The Kaplan-Meier sample average (KMSA) was used to account for the censoring issues, with costs and details discussed in the statistical analysis section. Both costs and effectiveness were discounted at the rate of $3 \%$ per year. ${ }^{24}$

\section{Covariates}

Patient demographics and tumor characteristics were included as baseline covariates to estimate the propensity scores. Covariates consisted of age at diagnosis, race/ethnicity, marital status, urban or rural area, SEER location, socioeconomic status, comorbidity, prostate cancer stage, Gleason score, and calendar year when cancer was diagnosed. Age at diagnosis was categorized into 5-year increments. Marital status was defined as married at time of diagnosis, not married, or unknown. Race/ethnicity was categorized as non-Hispanic white, nonHispanic black, Hispanic, and other. Urban area was defined as metropolitan counties and nonmetropolitan counties with a population of 20,000 or more, and rural contained nonmetropolitan counties with a population less than 20,000.

Charlson comorbidity at baseline was determined from Medicare inpatient and outpatient claims 12 months before diagnosis. ${ }^{15}$ We used the algorithm available on the National Cancer Institute's SEER-Medicare website, which assessed the following 14 conditions: acute myocardial infarction, history of myocardial infarction, congestive heart failure, peripheral vascular disease, cerebrovascular disease, chronic obstructive pulmonary disease, dementia, paralysis, any diabetes, chronic renal failure, any liver disease, ulcers, rheumatologic disease, and AIDS. The comorbidity index was weighted and then categorized as $0,1,2$, and $\geq 3$.

Tumor stage was identified using the American Joint Committee on Cancer (AJCC) 3rd edition for cases diagnosed before 2003 and derived from the AJCC 6th edition for cases diagnosed in 2004 and after. Cancer stage was categorized into stages III or IV. Gleason score was defined based on tumor grade and categorized as 2-4 (well differentiated), 5-7 (moderately differentiated), 8-10 (poorly differentiated or undifferentiated and anaplastic), and unknown.

\section{Statistical Analysis}

We examined the baseline characteristics of the study population across treatment groups using chi-squared tests. A propensity score method was used to control for pretreatment imbalances among the 3 groups. The propensity score was 
estimated by fitting logistic regression models with treatment status as the dependent variable, and the independent variables were patient characteristics at diagnosis, including age, race/ ethnicity, marital status, geographic location, SEER location, year of diagnosis, socioeconomic status, comorbidity, prostate cancer stage, and Gleason score. We used a 1:1 greedy-matching method with caliper of 0.05 to identify a 1:1 matched cohort of patients who received radiation versus radiation plus ADT and another 1:1 cohort of patients who received active surveillance versus radiation plus ADT. The common patients of both cohorts were identified to form a 1:1:1 matching cohort. The SAS macro \%GMATCH provided by the Mayo Clinic was used. After matching, the balance between matched groups was assessed by examining the standardized differences. We used the SAS macro \%STDDIFF developed by Yang et al. (2012) to estimate the standardized differences. ${ }^{25}$ The standardized differences were calculated for the comparisons of radiation plus ADT versus active surveillance and radiation plus ADT versus radiation only. The standardized difference represented the magnitude of the difference between groups and was independent of sample size.

The mean survival time with the corresponding 95\% confidence intervals (CIs) was estimated using Kaplan-Meier curves that took into consideration differential follow-up times. QALYs were calculated by weighting patients' survival-years and total health state utilities associated with each phase. QALYs were then discounted at the rate of 3\% annually.

In order to estimate lifetime costs, we calculated the monthly health care costs from prostate cancer diagnosis to death or end of study by using all claims for inpatient services, outpatient visits and procedures, skilled nursing facility use, home health care, and durable medical equipment. In order to account for censoring caused by incomplete follow-up, we used the KMSA method. The study period was partitioned into monthly intervals. For each monthly period, the average raw costs of patients who were still alive and not censored at the beginning of each period were estimated. These monthly costs were then weighted by multiplying by the overall probability of survival to that time. The weighted estimates were summed over all periods to obtain the mean censored adjusted costs. ${ }^{26}$ The lifetime costs were adjusted for inflation using the medical care component of the Consumer Price Index to 2016 U.S. dollars and, similar to QALYs, costs were discounted at 3\% annually.

The ICER was calculated as the difference in cost between 2 treatment groups divided by the difference in mean life-years or QALYs. We conducted one-way and probabilistic sensitivity analyses (PSAs) to evaluate how uncertainties affected the results. Health state utilities were not directly obtained from claims data and thus could be sources of variation. We accounted for these uncertainties in our analyses by alternately substituting health state utilities from worst- to best-case values. ICERs were calculated for each scenario. For PSAs, we used the bootstrap approach to repeatedly generate 5,000 samples. Cost-effectiveness acceptability curves were generated showing the proportion of bootstrap sample ICERs that fell below the willingness-to-pay threshold. The 95\% CIs of ICERs were also estimated from bootstrap samples. $P<0.05$ was considered significant.

\section{Results}

\section{Baseline Characteristics}

After applying the exclusion and inclusion criteria, we identified 8,001 eligible patients who underwent either (a) active surveillance, (b) radiation therapy, or (c) radiation plus ADT. The greedy-matching process resulted in a total of 2,802 patients with 934 patients in each treatment group. Table 2 and Table 3 show the baseline demographic and clinical characteristics with respect to treatment before and after matching. Before matching, all patient baseline characteristics except geographic location were significantly different $(P<0.01)$. Men aged $\geq 80$ years were more likely to choose active surveillance. Non-Hispanic black men were more likely to receive active surveillance, while non-Hispanic white men opted for more aggressive radiation plus ADT. Tumor clinical characteristics also varied across treatment groups. Men with a Gleason score of 8 or higher accounted for a higher proportion of patients treated with ADT and radiation (69.8\%) compared to $43.2 \%$ in the radiation alone and $57.9 \%$ in the active surveillance groups. Men who had clinical stage IV disease were more likely to receive radiation therapy plus ADT (53.1\%) and active surveillance (48.5\%). In general, radiation therapy plus ADT was more common among patients at higher risk of prostate cancer. After matching, the baseline characteristics of the 3 treatment groups were well balanced with all standardized differences $<0.15$.

\section{Costs, Life-Years Gained, and QALYs}

Table 4 and Table 5 illustrate the total life-year costs from a health care payer, effectiveness, and ICER perspective of the 3 treatment strategies. The mean lifetime health care costs (2016 U.S. dollars) were highest for the radiation plus ADT group $(\$ 177,704)$ followed by radiation therapy $(\$ 132,939)$ and active surveillance $(\$ 70,780)$. Further analysis of costs by phases showed that during the initial phase, the mean annual costs of active surveillance, radiation therapy, and radiation plus ADT were $\$ 8,902, \$ 27,345$, and $\$ 29,968$, respectively. The annual costs for the terminal phase were $\$ 50,096, \$ 80,613$, and $\$ 93,894$, respectively.

The mean life-years for the radiation plus ADT and radiation groups were 11.70 (95\% CI $=11.28-12.14)$ and 10.99 years (95\% CI $=10.56-11.42)$, respectively. Patients on active surveillance had the shortest mean life-years of 10.78 years (95\% $\mathrm{CI}=10.33-11.24)$. Adding ADT to radiation therapy increased mean patients' survival by 0.71 years (Table 4 ). 
TABLE 2 Demographic and Clinical Characteristics of Study Population by Treatment

\begin{tabular}{|c|c|c|c|c|c|c|c|}
\hline \multirow[b]{2}{*}{ Variables } & \multicolumn{2}{|c|}{$\begin{array}{l}\text { Active Surveillance } \\
\qquad(\mathrm{n}=1,672)\end{array}$} & \multicolumn{2}{|c|}{$\begin{array}{l}\text { Radiation } \\
(\mathrm{n}=1,111)\end{array}$} & \multicolumn{2}{|c|}{$\begin{array}{l}\text { Radiation plus ADT } \\
(\mathrm{n}=5,218)\end{array}$} & \multirow[b]{2}{*}{$P$ Value } \\
\hline & $\mathrm{n}$ & $\%$ & $\mathrm{n}$ & $\%$ & $\mathrm{n}$ & $\%$ & \\
\hline \multicolumn{8}{|l|}{ Age, years } \\
\hline $66-69$ & 511 & 30.56 & 278 & 25.02 & 1,229 & 23.55 & $<0.01$ \\
\hline $70-74$ & 457 & 27.33 & 417 & 37.53 & 1,761 & 33.75 & \\
\hline $75-79$ & 345 & 20.63 & 277 & 24.93 & 1,330 & 25.49 & \\
\hline $80+$ & 359 & 21.47 & 139 & 12.51 & 898 & 17.21 & \\
\hline \multicolumn{8}{|l|}{ Race } \\
\hline Non-Hispanic white & 1,149 & 68.72 & 850 & 76.51 & 4,214 & 80.76 & $<0.01$ \\
\hline Non-Hispanic black & 360 & 21.53 & 148 & 13.32 & 469 & 8.99 & \\
\hline Hispanic & 102 & 6.10 & 43 & 3.87 & 304 & 5.83 & \\
\hline Other & 61 & 3.65 & 70 & 6.30 & 231 & 4.43 & \\
\hline \multicolumn{8}{|l|}{ Marital status } \\
\hline Unmarried & 598 & 35.77 & 281 & 25.29 & 1,194 & 22.88 & $<0.01$ \\
\hline Married & 955 & 57.12 & 768 & 69.13 & 3,711 & 71.12 & \\
\hline Unknown & 119 & 7.12 & 62 & 5.58 & 313 & 6.00 & \\
\hline \multicolumn{8}{|l|}{ Geographic location } \\
\hline Rural & 165 & 9.87 & 89 & 8.01 & 517 & 9.91 & 0.14 \\
\hline Urban & 1,507 & 90.13 & 1,022 & 91.99 & 4,701 & 90.09 & \\
\hline \multicolumn{8}{|l|}{ SEER location } \\
\hline Midwest & 296 & 17.70 & 289 & 26.01 & 1,113 & 21.33 & $<0.01$ \\
\hline Northeast & 235 & 14.06 & 167 & 15.03 & 964 & 18.47 & \\
\hline South & 219 & 13.10 & 107 & 9.63 & 610 & 11.69 & \\
\hline West & 922 & 55.14 & 548 & 49.32 & 2,531 & 48.51 & \\
\hline \multicolumn{8}{|l|}{ SES median income (in quartiles) } \\
\hline lst quartile $(\leq \$ 30,028)$ & 565 & 33.79 & 329 & 29.61 & 1,107 & 21.22 & $<0.01$ \\
\hline 2nd quartile $(\$ 30,029-\$ 40,618)$ & 436 & 26.08 & 285 & 25.65 & 1,279 & 24.51 & \\
\hline 3rd quartile $(\$ 40,619-\$ 55,962)$ & 377 & 22.55 & 270 & 24.30 & 1,354 & 25.95 & \\
\hline 4th quartile $(>\$ 55,962)$ & 294 & 17.58 & 227 & 20.43 & 1,478 & 28.33 & \\
\hline \multicolumn{8}{|l|}{ Comorbidity score } \\
\hline 0 & 1,368 & 81.82 & 843 & 75.88 & 3,848 & 73.75 & $<0.01$ \\
\hline 1 & 185 & 11.06 & 177 & 15.93 & 890 & 17.06 & \\
\hline 2 & 66 & 3.95 & 55 & 4.95 & 312 & 5.98 & \\
\hline$\geq 3$ & 53 & 3.17 & 36 & 3.24 & 168 & 3.22 & \\
\hline \multicolumn{8}{|l|}{ Prostate cancer stage } \\
\hline Stage III & 861 & 51.50 & 732 & 65.89 & 2,450 & 46.95 & $<0.01$ \\
\hline Stage IV & 811 & 48.50 & 379 & 34.11 & 2,768 & 53.05 & \\
\hline \multicolumn{8}{|l|}{ Gleason score } \\
\hline $2-4$ (grade 1$)$ & 53 & 3.17 & 63 & 5.67 & 69 & 1.32 & $<0.01$ \\
\hline 5-7 (grade 2) & 651 & 38.94 & 568 & 51.13 & 1,507 & 28.88 & \\
\hline $8-10$ (grade 3$)$ & 968 & 57.89 & 480 & 43.20 & 3,642 & 69.80 & \\
\hline \multicolumn{8}{|l|}{ Year of diagnosis } \\
\hline 1992-1996 & 601 & 35.94 & 608 & 54.73 & 1,206 & 23.11 & $<0.01$ \\
\hline $\begin{array}{l}1997-2001 \\
\end{array}$ & 331 & 19.80 & 180 & 16.20 & 1,336 & 25.60 & \\
\hline $2002-2006$ & 420 & 25.12 & 198 & 17.82 & 1,729 & 33.14 & \\
\hline $2007-2009$ & 320 & 19.14 & 125 & 11.25 & 947 & 18.15 & \\
\hline
\end{tabular}

There was no statistically significant difference in QALYs across treatment groups $(P=0.36)$. Patients on active surveillance had mean QALYs of 8.75 (95\% CI=8.35-9.15) compared with 8.56 (95\% CI=8.19-8.92) and 8.91 (95\% CI=8.56-9.26) for the radiation and radiation plus ADT groups, respectively (Table 5).
The ICER for radiation plus ADT versus radiation alone was $\$ 63,049$ per mean life-year gained and for radiation compared with active surveillance was $\$ 295,995$ per mean life-year gained (Table 4). As expected, treatment-associated adverse side effects substantially affected QALYs gained. Compared 
TABLE 3 Baseline Characteristics of Patients After Individual Greedy Matching ( $\mathrm{n=2,829)}$

\begin{tabular}{|c|c|c|c|c|c|c|c|c|c|}
\hline \multirow[b]{3}{*}{ Variables } & \multirow{2}{*}{\multicolumn{2}{|c|}{$\begin{array}{l}\text { Active Surveillance } \\
\qquad(\mathrm{n}=943)\end{array}$}} & \multirow{2}{*}{\multicolumn{2}{|c|}{$\begin{array}{c}\text { Radiation } \\
(\mathrm{n}=943)\end{array}$}} & \multirow{2}{*}{\multicolumn{2}{|c|}{$\begin{array}{c}\text { Radiation + ADT } \\
(\mathrm{n}=943)\end{array}$}} & \multicolumn{2}{|c|}{ Standardized Difference } & \multirow[b]{3}{*}{$P$ Value } \\
\hline & & & & & & & \multirow{2}{*}{\begin{tabular}{|c|} 
Active \\
Surveillance \\
vs. Radiation \\
plus ADT \\
\end{tabular}} & \multirow{2}{*}{$\begin{array}{c}\text { Radiation } \\
\text { vs. Radiation } \\
\text { plus ADT }\end{array}$} & \\
\hline & $\mathrm{n}$ & $\%$ & $\mathrm{n}$ & $\%$ & $\mathrm{n}$ & $\%$ & & & \\
\hline \multicolumn{10}{|l|}{ Age, years } \\
\hline $66-69$ & 264 & 28.00 & 249 & 26.41 & 240 & 25.45 & 0.04 & 0.06 & 0.70 \\
\hline $70-74$ & 324 & 34.36 & 324 & 34.36 & 340 & 36.06 & & & \\
\hline $75-79$ & 220 & 23.33 & 234 & 24.81 & 242 & 25.66 & & & \\
\hline $80+$ & 135 & 14.32 & 136 & 14.42 & 121 & 12.83 & & & \\
\hline \multicolumn{10}{|l|}{ Race } \\
\hline Non-Hispanic white & 705 & 74.76 & 713 & 75.61 & 762 & 80.81 & 0.03 & 0.13 & 0.05 \\
\hline Non-Hispanic black & 145 & 15.38 & 135 & 14.32 & 108 & 11.45 & & & \\
\hline Hispanic & 42 & 4.45 & 42 & 4.45 & 28 & 2.97 & & & \\
\hline Others & 51 & 5.41 & 53 & 5.62 & 45 & 4.77 & & & \\
\hline \multicolumn{10}{|l|}{ Marital status } \\
\hline Unmarried & 265 & 28.10 & 261 & 27.68 & 241 & 25.56 & 0.04 & 0.05 & 0.55 \\
\hline Married & 610 & 64.69 & 623 & 66.07 & 643 & 68.19 & & & \\
\hline Unknown & 68 & 7.21 & 59 & 6.26 & 59 & 6.26 & & & \\
\hline \multicolumn{10}{|l|}{ Geographic location } \\
\hline Rural & 68 & 7.21 & 82 & 8.70 & 88 & 9.33 & -0.05 & -0.02 & 0.23 \\
\hline Urban & 875 & 92.79 & 861 & 91.30 & 855 & 90.67 & & & \\
\hline \multicolumn{10}{|l|}{ SEER location } \\
\hline Midwest & 203 & 21.53 & 208 & 22.06 & 227 & 24.07 & 0.02 & 0.08 & 0.59 \\
\hline Northeast & 141 & 14.95 & 140 & 14.85 & 148 & 15.69 & & & \\
\hline South & 100 & 10.60 & 96 & 10.18 & 79 & 8.38 & & & \\
\hline West & 499 & 52.92 & 499 & 52.92 & 489 & 51.86 & & & \\
\hline \multicolumn{10}{|l|}{ SES median income (in quartiles) } \\
\hline lst quartile $(\leq \$ 30,028)$ & 278 & 29.48 & 284 & 30.12 & 254 & 26.94 & 0.02 & 0.08 & 0.78 \\
\hline 2nd quartile $(\$ 30,029-\$ 40,618)$ & 248 & 26.30 & 239 & 25.34 & 252 & 26.72 & & & \\
\hline 3rd quartile $(\$ 40,619-\$ 55,962)$ & 223 & 23.65 & 232 & 24.60 & 232 & 24.60 & & & \\
\hline 4th quartile $(>\$ 55,962)$ & 194 & 20.57 & 188 & 19.94 & 205 & 21.74 & & & \\
\hline \multicolumn{10}{|l|}{ Comorbidity score } \\
\hline 0 & 741 & 78.58 & 740 & 78.47 & 731 & 77.52 & 0.02 & 0.08 & 0.97 \\
\hline 1 & 122 & 12.94 & 127 & 13.47 & 135 & 14.32 & & & \\
\hline 2 & 48 & 5.09 & 43 & 4.56 & 48 & 5.09 & & & \\
\hline$\geq 3$ & 32 & 3.39 & 33 & 3.50 & 29 & 3.08 & & & \\
\hline \multicolumn{10}{|l|}{ Prostate cancer stage } \\
\hline Stage III & 584 & 61.93 & 587 & 62.25 & 585 & 62.04 & 0.02 & 0.08 & 0.99 \\
\hline Stage IV & 359 & 38.07 & 356 & 37.75 & 358 & 37.96 & & & \\
\hline \multicolumn{10}{|l|}{ Gleason score } \\
\hline $2-4$ (grade 1$)$ & 45 & 4.77 & 34 & 3.61 & 32 & 3.39 & 0.06 & 0.01 & 0.57 \\
\hline 5-7 (grade 2) & 452 & 47.93 & 467 & 49.52 & 464 & 49.20 & & & \\
\hline 8 -10 (grade 3) & 446 & 47.30 & 442 & 46.87 & 447 & 47.40 & & & \\
\hline \multicolumn{10}{|l|}{ Year of diagnosis } \\
\hline $1992-1996$ & 471 & 49.95 & 467 & 49.52 & 466 & 49.42 & 0.04 & 0.14 & 0.09 \\
\hline $1997-2001$ & 172 & 18.24 & 161 & 17.07 & 206 & 21.85 & & & \\
\hline $2002-2006$ & 181 & 19.19 & 193 & 20.47 & 176 & 18.66 & & & \\
\hline $2007-2009$ & 119 & 12.62 & 122 & 12.94 & 95 & 10.07 & & & \\
\hline
\end{tabular}

with radiation only, the incremental cost of radiation plus ADT was $\$ 127,900$ per mean QALY. Radiation only versus active surveillance was dominated as it incurred significant incremental costs but offered no improvement in QALYs (Table 5).

\section{Sensitivity Analysis}

When we re-ran the analysis assuming the worst-case and best-case health state utilities from the literature, radiation plus ADT versus radiation only had an ICER ranging from 


\begin{tabular}{|c|c|c|c|c|c|}
\hline Treatment Groups & $\begin{array}{c}\text { Life-Years } \\
(95 \% \text { CI })\end{array}$ & $\begin{array}{c}\text { Censored Adjusted Total } \\
\text { Costs (\$US 2016) } \\
\text { Mean/Median (SE) } \\
\end{array}$ & Incremental Life-Years & Incremental Costs & $\begin{array}{l}\text { ICER per Life-Year } \\
\text { Gaineda }(95 \% \text { CI) }\end{array}$ \\
\hline \multicolumn{6}{|c|}{ Mean life-year and ICER per mean life-year gained } \\
\hline Active surveillance & $\begin{array}{c}10.78 \\
(10.33-11.24)\end{array}$ & $\begin{array}{c}\$ 70,780 / \$ 22,192 \\
(121,154)\end{array}$ & N/A & N/A & N/A \\
\hline Radiation & $\begin{array}{c}10.99 \\
(10.56-11.42)\end{array}$ & $\begin{array}{c}\$ 132,939 / \$ 79,200 \\
(155,023)\end{array}$ & 0.21 & $\$ 62,159$ & $\begin{array}{c}295,995 \\
(-130,336-1,124,453)^{\mathrm{b}} \\
\text { Weakly dominated }\end{array}$ \\
\hline Radiation plus ADT & $\begin{array}{c}11.70 \\
(11.28-12.14) \\
\end{array}$ & $\begin{array}{c}\$ 177,704 / \$ 119,611 \\
(186,816)\end{array}$ & 0.71 & $\$ 44,765$ & $\begin{array}{c}63,049 \\
(37,049-161,213)^{c} \\
\end{array}$ \\
\hline \multicolumn{6}{|c|}{$\begin{array}{l}\text { aThe ICER was calculated as the difference in cost between } 2 \text { treatment groups divided by the difference in life-years. } \\
\text { bICER for the comparison between radiation only vs. active surveillance. Compared with active surveillance, radiation only was weakly dominated, since it incurred } \\
\text { significant incremental costs but yielded minimal incremental life-years. } \\
\text { cICER for the comparison between radiation + ADT vs. radiation only. }\end{array}$} \\
\hline
\end{tabular}

$\$ 131,662$ per QALY to $\$ 78,535$ per QALY, respectively. As the health state utilities ranged from the worst to the best scenario, QALYs grew closer to the life-years and thus ICERs declined. Sensitivity analyses showed that the higher range of health state utilities was associated with lower cost per QALY. Radiation versus active surveillance was dominated, as radiation incurred significant incremental costs but no improvement in incremental life-years, and the ICER was $\$ 388,494$ per QALY in the best-case scenario.

The cost-effectiveness acceptability curves in Appendix A and Appendix B (available in online article) show the probability (vertical axis) that each strategy was cost-effective as the willingnessto-pay for a life-year or a QALY rose along the horizontal axis. At the willingness-to-pay threshold of approximately $\$ 50,000$ per life-year, the probability that radiation plus ADT was costeffective compared with radiation alone was about $50 \%$. The probability that radiation plus ADT was cost-effective increased to more than $80 \%$ as the threshold increased to $\$ 80,000$ per lifeyear. Compared with active surveillance, radiation plus ADT was $50 \%$ and $80 \%$ cost-effective at the thresholds of $\$ 110,000$ and $\$ 170,000$ per life-year, respectively. As the threshold continued to increase, both comparisons became similar until they reached $100 \%$. As expected, radiation plus ADT was less likely to be a cost-effective strategy when taking into account the effects of its associated AEs. In Appendix B, radiation plus ADT was nearly $80 \%$ cost-effective at a willingness-to-pay threshold of $\$ 210,000$ per QALY compared with radiation alone.

\section{Discussion}

Our analysis shows that ADT plus radiation therapy substantially improved survival and QALYs at reasonable costs. Under base-case conditions, ADT plus radiation costs about $\$ 63,049$ per life-year gained (2016 U.S. dollars) from the health care payer perspective compared with radiation alone. The ICER comparing ADT plus radiation and radiation only increased to
$\$ 127,900$ per QALY when considering all potentially rare AEs related to ADT. According to the World Health Organization, an intervention is considered very cost-effective if it costs less than the GDP per capita for each QALY and cost-effective if it costs 1-3 times the GDP per capita. ${ }^{27}$ The U.S. GDP per capita was $\$ 57,467$ in 2016 , and by either standard, radiation plus ADT remained a cost-effective intervention from the health care payer perspective compared with radiation alone.

Survival benefits, treatment-related side effects, and costs are all important considerations in selecting appropriate prostate cancer therapy. Previous economic evaluations of hormonal therapy for prostate cancer patients used Markov models with transitional health states to model disease progression. Most analyses of ADT were published before concerns about its long-term side effects were raised. ${ }^{12,13}$ Two studies conducted in the United States had radiotherapy as the baseline treatment strategy. ${ }^{10,11}$ In both studies, Konski et al. (2005, 2006) estimated model parameters from a single clinical trial. ${ }^{10,11}$ In the first study, they found that radiation plus hormone therapy for 4 months was cost-effective compared with radiation alone at $\$ 2,153$ per QALY (2003 U.S. dollars). ${ }^{10}$ In a subsequent study, they extended the analysis to compare 2 years of long-term use of ADT plus radiation versus short-term adjuvant ADT and reported a cost-effectiveness ratio of $\$ 4,605$ per QALY. ${ }^{11}$ Our results were not directly comparable because of differences in study population and cost estimation method. While Konski used prostate cancer-specific costs, we estimated patients' total lifetime costs, which included all costs associated with care and treatment of side effects, if any. Our population reported more variation of ADT duration (interquartile range $=6.7-43.5$ months). In addition to the discomforts that affect men's QoL during the initial phase of treatment, unlike previous studies, we took into account the long-term complications associated with ADT use during the continuing phase. 
Cost-Effectiveness of Adding Androgen Deprivation Therapy to Radiation

Therapy for Men with Advanced Prostate Cancer from a U.S. Payer's Perspective

TABLE 5 QALYs and Cost-Effectiveness by Treatment Group

\begin{tabular}{|c|c|c|c|c|c|}
\hline Treatment Groups & $\begin{array}{l}\text { QALYs } \\
(95 \% \mathrm{CI})\end{array}$ & $\begin{array}{c}\text { Censored Adjusted Total } \\
\text { Costs (\$) } \\
\text { Mean/Median (SE) }\end{array}$ & Incremental QALYs & Incremental Costs & $\begin{array}{l}\text { ICER per QALYa } \\
(95 \% \mathrm{CI})\end{array}$ \\
\hline \multicolumn{6}{|c|}{ Mean QALYs and ICER per mean QALY } \\
\hline Active surveillance & $\begin{array}{c}8.75 \\
(8.35-9.15)\end{array}$ & $\begin{array}{c}\$ 70,780 / \$ 22,192 \\
(121,154)\end{array}$ & N/A & N/A & N/A \\
\hline Radiation & $\begin{array}{c}8.56 \\
(8.19-8.92) \\
\end{array}$ & $\begin{array}{c}\$ 132,939 / \$ 79,200 \\
(155,023)\end{array}$ & -0.19 & $\$ 62,159$ & Dominated $^{b}$ \\
\hline Radiation + ADT & $\begin{array}{c}8.91 \\
(8.56-9.26)\end{array}$ & $\begin{array}{c}\$ 177,704 / \$ 119,611 \\
(186,816)\end{array}$ & 0.35 & $\$ 44,765$ & $\begin{array}{c}127,900^{c} \\
(55,009-621,039)\end{array}$ \\
\hline \multicolumn{6}{|c|}{$\begin{array}{l}\text { aThe ICER was calculated as the difference in cost between } 2 \text { treatment groups divided by the difference in QALYs. } \\
\text { bICER for the comparison between radiation only vs. active surveillance. Compared with active surveillance, radiation only was dominated, since it incurred significant } \\
\text { incremental costs but no improvement in QALYs. } \\
\text { 'ICER for the comparison between radiation }+ \text { ADT vs. radiation only. } \\
\text { ADT = androgen deprivation therapy; CI = confidence interval; ICER=incremental cost-effectiveness ratio; N/A = not applicable; QALY=quality-adjusted life-year; } \\
\text { SE=standard error. }\end{array}$} \\
\hline
\end{tabular}

The average lifetime costs of prostate cancer patients were estimated at $\$ 70,780$ for active surveillance, $\$ 132,939$ for radiation, and $\$ 177,704$ for the radiation plus ADT group (2016 U.S. dollars). The figures were consistent with the literature. The most recent study reported that the lifetime cost of care for prostate cancer patients among the Medicare population was $\$ 110,520 .{ }^{28}$ Estimating lifetime costs using patient-level data was challenging as costs were censored and those incurred after the censoring date were unknown. We carefully adjusted costs for censoring using the KMSA method. The method has been considered as a reasonable approach for dealing with censoring, and it performs well over different levels of censoring. ${ }^{29}$

In our study, utilities were considered as potential sources of variation. We conducted a sensitivity analysis to explore the effects of health states utilities on ICERs and presented results for worst- and best-case scenarios. Valuing treatment discomforts and side effects at the worst-case scenario made radiation plus ADT and radiation alone become less cost-effective compared with active surveillance. The ICER of radiation plus ADT versus radiation only changed by approximately 10\%-40\% when changing the utilities from the worst-case to the bestcase scenario, showing the effects of utility uncertainty.

This is the first economic evaluation that comprehensively assessed major long-term side effects associated with ADT among the Medicare population. The strength of this study is its use of a population-based SEER-Medicare database that was well suited to study long-term and even rare AEs. The population coverage was high, with an estimated $96 \%$ of adults aged $\geq 65$ years enrolled in Medicare. The study used propensity scorematching to control for potential confounders. The use of propensity score-matching allowed the assessment of covariate balance and could help to reduce selection bias due to patients choosing a particular treatment strategy according to their baseline characteristics. The common-reference group 1:1 approach used in our study to match radiation plus ADT versus radiation only and radiation only versus active surveillance was not as ideal as three-way 1:1:1 matching. However, the common-reference group 1:1 approach significantly reduced bias and was comparable to other matching methods. ${ }^{30}$ The propensity score method had its own limitation, since it was unable to account for unmeasured confounders that influenced the treatment assignment.

\section{Limitations}

Our study has limitations to consider. The study population reflected the elderly patient population and practice patterns in the United States, which were likely different from those of other countries. The findings may not be generalized to patients aged $<65$ years. Economic analysis was performed from the U.S. payer perspective based on Medicare insurance payments. Other indirect costs relating to patients' out-ofpocket expenses and caregivers were omitted. We did not take into account the cancer recurrence status, which might affect patients' QoL. In addition, there was a substantial lag time of data for prostate cancer cases in 1992-2009; updated analyses for more recent cases will be needed in the near future.

\section{Conclusions}

Despite ADT-associated costs and long-term side effects, this study indicated that radiation plus ADT continued to be the cost-effective therapy at $\$ 63,049$ per life-year and $\$ 127,900$ per QALY compared with radiation alone. The findings should be helpful to clinical oncologists and policymakers in choosing the most cost-effective treatment modalities for patients with prostate cancer by considering long-term complications associated with ADT use during the various phases of care. 


\section{Authors}

CHI NGUYEN, PhD, and XIANGLIN L. DU, MD, PhD, Department of Epidemiology, Human Genetics, and Environmental Science;

DAVID R. LAIRSON, PhD, Department of Management Policy and Community Health; and MICHAEL D. SWARTZ, PhD, Department of Biostatistics and Data Science, School of Public Health, University of Texas Health Science Center at Houston, Texas.

AUTHOR CORRESPONDENCE: Xianglin L. Du, MD, PhD, University of Texas School of Public Health, 1200 Pressler St., RAS-E631, Houston, TX 77030.E-mail:Xianglin.L.Du@uth.tmc.edu.

\section{DISCLOSURES}

This research was supported in part by the Cancer Prevention Research Institute of Texas (grant nos. RP130051 and RP170668). The authors declare that there are no conflicts of interest.

\section{ACKNOWLEDGMENTS}

We acknowledge the efforts of the National Cancer Institute; Centers for Medicare \& Medicaid Services; Information Management Services; and the Surveillance, Epidemiology, and End Results Program tumor registries in the creation of this database. The interpretation and reporting of these data are the sole responsibilities of the authors.

\section{REFERENCES}

1. Golabek T, Belsey J, Drewa T, et al. Evidence-based recommendations on androgen deprivation therapy for localized and advanced prostate cancer. Cent European J Urol. 2016;69(2):131-38.

2. Shahinian VB, Kuo YF, Gilbert SM. Reimbursement policy and androgendeprivation therapy for prostate cancer. N Engl J Med. 2010;363(19):1822-32.

3. Shahinian VB, Kuo YF, Freeman JL, Goodwin JS. Risk of fracture after androgen deprivation for prostate cancer. N Engl J Med. 2005;352(2):154-64.

4. Saigal CS, Gore JL, Krupski TL, Hanley J, Schonlau M, Litwin MS; Urologic Diseases in America Project. Androgen deprivation therapy increases cardiovascular morbidity in men with prostate cancer. Cancer. 2007;110(7):1493-500

5. Ehdaie B, Atoria CL, Gupta A, et al. Androgen deprivation and thromboembolic events in men with prostate cancer. Cancer. 2012;118(13):3397-406.

6. Keating NL, O'Malley AJ, Freedland SJ, Smith MR. Diabetes and cardiovascular disease during androgen deprivation therapy: observational study of veterans with prostate cancer. J Natl Cancer Inst. 2010;102(1):39-46.

7. Keating NL, O'Malley AJ, Smith MR. Diabetes and cardiovascular disease during androgen deprivation therapy for prostate cancer. J Clin Oncol. 2006;24(27):4448-56.

8. Snyder CF, Frick KD, Blackford AL, et al. How does initial treatment choice affect short-term and long-term costs for clinically localized prostate cancer? Cancer. 2010;116(23):5391-99.

9. Hatoum HT, Crawford ED, Nielsen SK, Lin SJ, Marshall DC. Review of the economic evaluations of hormonal therapy for patients with locally advanced prostate cancer. Expert Rev Pharmacoecon Outcomes Res. 2013;13(2):251-59.

10. Konski A, Sherman E, Krahn M, et al. Economic analysis of a phase III clinical trial evaluating the addition of total androgen suppression to radiation versus radiation alone for locally advanced prostate cancer (Radiation Therapy Oncology Group protocol 86-10). Int J Radiat Oncol Biol Phys. 2005;63(3):788-94
11. Konski A, Watkins-Bruner D, Brereton H, Feigenberg S, Hanks G. Longterm hormone therapy and radiation is cost-effective for patients with locally advanced prostate carcinoma. Cancer. 2006;106(1):51-57.

12. Hillner BE, McLeod DG, Crawford ED, Bennett CL. Estimating the cost effectiveness of total androgen blockade with flutamide in $\mathrm{Ml}$ prostate cancer. Urology. 1995;45(4):633-40.

13. Bayoumi AM, Brown AD, Garber AM. Cost-effectiveness of androgen suppression therapies in advanced prostate cancer. J Natl Cancer Inst. 2000;92(21):1731-39.

14. Hayes JH, Ollendorf DA, Pearson SD, et al. Observation versus initial treatment for men with localized, low-risk prostate cancer: a cost-effectiveness analysis. Ann Intern Med. 2013;158(12):853-60.

15. National Cancer Institute. SEER-Medicare linked data. Available at: https:// healthcaredelivery.cancer.gov/seermedicare/. Accessed December 12, 2018.

16. Thein HH, Qiao Y, Zaheen A, et al. Cost-effectiveness analysis of treatment with non-curative or palliative intent for hepatocellular carcinoma in the real-world setting. PLoS One. 2017;12(10):e0185198.

17. Stewart ST, Lenert L, Bhatnagar V, Kaplan RM. Utilities for prostate cancer health states in men aged 60 and older. Med Care. 2005;43(4):347-55.

18. Roth JA, Gulati R, Gore JL, Cooperberg MR, Etzioni R. Economic analysis of prostate-specific antigen screening and selective treatment strategies, JAMA Oncol. 2016;2(7):890-98

19. Heijnsdijk EA, de Carvalho TM, Auvinen A, et al. Cost-effectiveness of prostate cancer screening: a simulation study based on ERSPC data. J Natl Cancer Inst. 2015;107(1):366.

20. Hiligsmann M, Ethgen O, Richy F, Reginster JY. Utility values associated with osteoporotic fracture: a systematic review of the literature. Calcif Tissue Int. 2008;82(4):288-92

21. Chowdhury EK, Ademi Z, Moss JR, et al. Cost-utility of angiotensin-converting enzyme inhibitor-based treatment compared with thiazide diureticbased treatment for hypertension in elderly Australians considering diabetes as comorbidity. Medicine (Baltimore). 2015;94(9):e590.

22. Hay JW, Sterling KL. Cost effectiveness of treating low HDL-cholesterol in the primary prevention of coronary heart disease. Pharmacoeconomics. 2005;23(2):133-41.

23. Yu SY, Lee TJ, Jang SH, Han JW, Kim TH, Kim KW. Cost-effectiveness of nationwide opportunistic screening program for dementia in South Korea. J Alzheimers Dis. 2015;44(1):195-204.

24. Neumann PJ, Sanders GD, Russell LB, Siegel JE, Ganiats TG. CostEffectiveness in Health and Medicine. 2nd ed. New York: Oxford University Press; 2016.

25. Yang D, Dalton JE. A unified approach to measuring the effect size between two groups using SAS. Paper 335-2012. Presented at: SAS Global Forum; April 22-25, 2012; Orlando, FL.

26. Gray AM, Clark PM, Wolstenholme JL, Wordsworth S. Applied Methods of Cost-effectiveness Analysis in Health Care. New York: Oxford University Press; 2011

27. Marseille E, Larson B, Kazi DS, Kahn JG, Rosen S. Thresholds for the cost-effectiveness of interventions: alternative approaches. Bull World Health Organ. 2015;93(2):118-24.

28. Stokes ME, Ishak J, Proskorovsky I, Black LK, Huang Y. Lifetime economic burden of prostate cancer. BMC Health Serv Res. 2011;11:349.

29. O'Hagan A, Stevens JW. On estimators of medical costs with censored data. J Health Econ. 2004;23(3):615-25.

30. Rassen JA, Shelat AA, Franklin JM, Glynn RJ, Solomon DH, Schneeweiss S. Matching by propensity score in cohort studies with three treatment groups. Epidemiology. 2013;24(3):401-09. 
Cost-Effectiveness of Adding Androgen Deprivation Therapy to Radiation

\section{APPENDIX A Cost-Effectiveness Acceptability Curves of Life-Years}

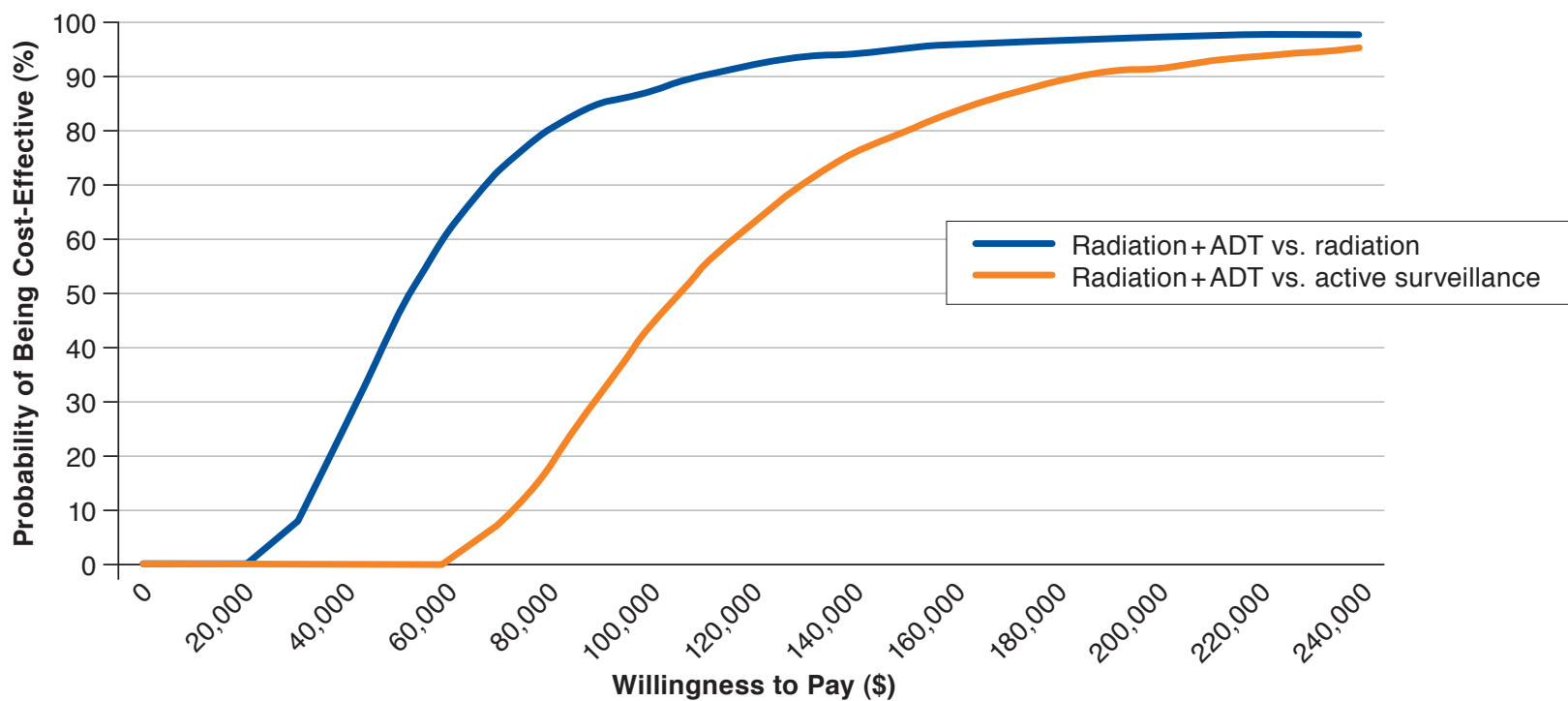

APPENDIX B Cost-Effectiveness Acceptability Curves of OALYs

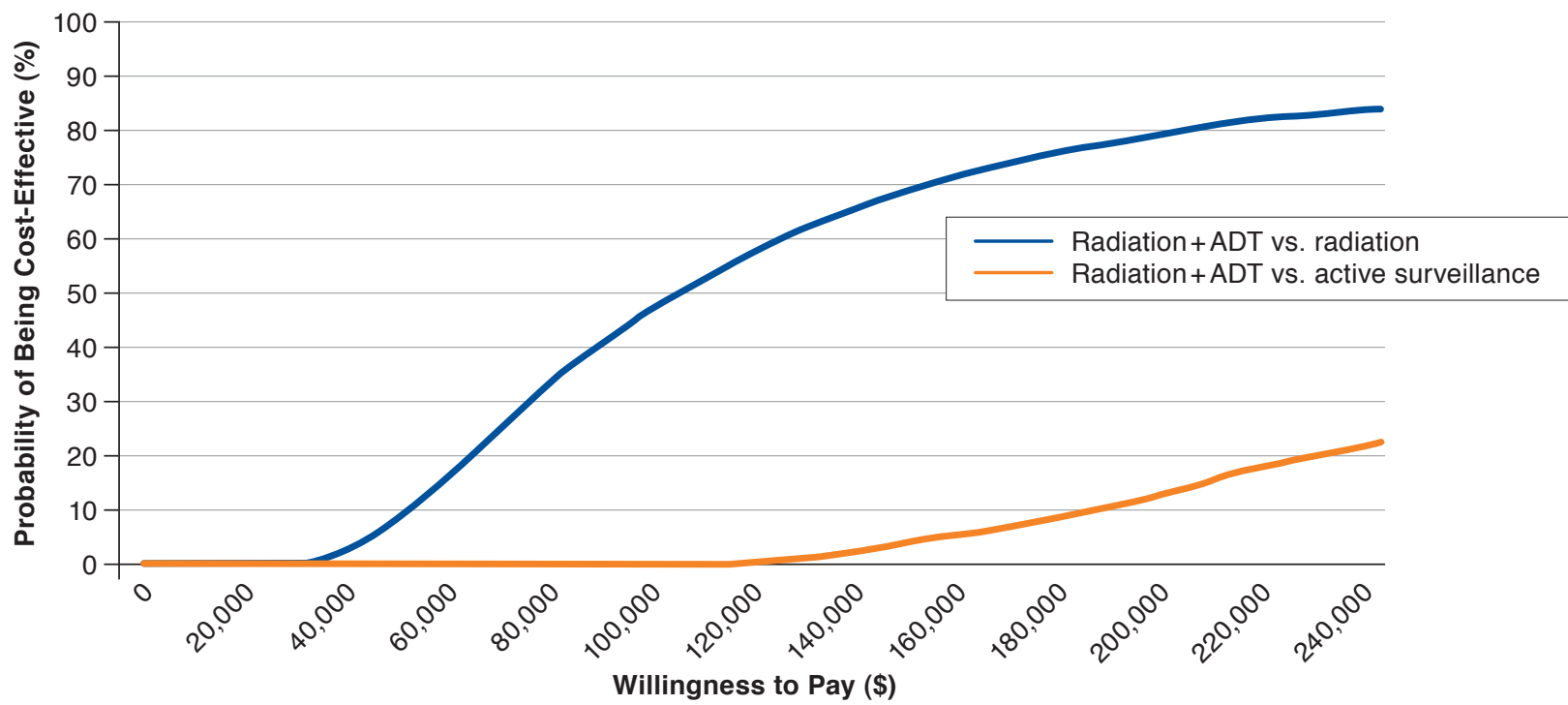

$A D T=$ androgen deprivation therapy; $Q A L Y=$ quality-adjusted life-year 